\title{
Épaisseur d'os alvéolaire dans la zone du point A : savoir éviter les échecs parodontaux en regard des incisives maxillaires
}

\author{
Émilie CHEVALIER ${ }^{1 *}$, Camille PHILIP-ALLIEZ ${ }^{2}$, Michel LE GALL ${ }^{1,2}$ \\ 1 Villa Gabrielle, 314 boulevard Marcel Pagnol, 13400 Aubagne, France \\ 2 Unité Fonctionnelle d’Orthopédie Dento-Faciale, Hôpital de la Timone Marseille, 264 Rue Saint-Pierre, 13385 Marseille Cedex 5 , \\ France
}

MOTS CLÉS :

Épaisseur d'os alvéolaire /

Résorption osseuse /

Tomodensitométrie

\section{KEYWORDS:}

Alveolar bone thickness / Bone resorption /

CT scan
RÉSUMÉ - Nombreuses sont les études sur les relations orthodontie/parodontie mais rares sont celles qui ont bénéficié de l'apport des nouvelles techniques d'imageries 3D et qui mettent en avant les effets iatrogènes que peut avoir l'orthodontie sur le parodonte. Les risques parodontaux en terme de fenestrations, de déhiscences osseuses en regard des incisives maxillaires sont réels pendant ou après un traitement orthodontique. D'ou l'importance d'évaluer précisément la situation initiale en terme de quantité d'os dans ce secteur esthétique d'arcade. Notre étude a eu pour objectif d'évaluer la fiabilité de la téléradiographie de profil conventionnelle pour quantifier l'épaisseur d'os alvéolaire en regard des incisives maxillaires en la comparant aux données issues du scanner. L'objectif second était de mettre en évidence un profil de patient dit «à risque » en évaluant les éventuelles corrélations entre cette épaisseur et des composantes de dysmorphie. Les résultats ont révélé une erreur d'évaluation de moitié quant à l'estimation de l'épaisseur d'os et un risque accru en cas d'hyperdivergence, de classe III et de proalvéolie. Enfin, compte tenu de ces résultats, nous avons abordé les conduites à tenir cliniquement pour éviter l'apparition de tels échecs parodontaux dans ce secteur d'arcade.

ABSTRACT - Alveolar bone thickness in A point area : how to avoid periodontal failures in front of upper incisors. Studies on orthodontic-periodontics relationships are numerous but few have benefited from the contribution of new 3D imaging techniques that emphasize iatrogenic effects that orthodontics may have on the periodontium. Periodontal risk in terms of fenestration, bone dehiscences next maxillary incisors are real during or after orthodontic treatment. The accurate assessment of the initial situation in terms of bone quantity in this dental arch anterior segment is thus very important. Our study aimed to evaluate the reliability of conventional lateral cephalograms to quantify alveolar bone thickness in relation to the maxillary incisors by comparing it with data from CT scans. The second objective was to identify an at risk patient profile by assessing possible correlations between this thickness and dysmorphia components. The results revealed a half of assessment error in the estimation of bone thickness and increased risk in case of hyperdivergence typology, Class III skeletal relationships and dento-alveolar protrusion. Finally, in view of these data, we discussed the clinical procedures to avoid such periodontal failures in this anterior segment of the dental arch.

\footnotetext{
*Auteur pour correspondance : emilie_cassis@hotmail.com
} 


\section{Introduction}

Le parodonte est l'unité physiologique commune aux deux disciplines que sont l'orthodontie et la parodontie. Si les apports bénéfiques de l'orthodontie à la parodontie sont souvent rapportés dans la littérature (aménagement des sites parodontaux périimplantaire, restauration de l'espace biologique, réarrangement des collets, correction des migrations secondaires, facilitation du contrôle de plaque et de l'hygiène bucco-dentaire), les effets iatrogènes de l'orthodontie sur le parodonte le sont beaucoup moins et méritent que l'on s'y intéresse pour mieux les appréhender et essayer de les éviter. Toute détérioration du support parodontal à plus ou moins long terme consécutivement à un traitement d'orthodontie constitue un échec thérapeutique.

La zone du point A anatomiquement complexe est une zone dont il est nécessaire de connaître les particularités lorsque l'on envisage un déplacement dentaire sur les dents antérieures.

Utilisé par presque toutes les analyses populaires, le point A défini par Downs sert, entre autres, de point de référence pour l'analyse des compensations dento-alvéolaires (analyses de Tweed, Steiner, Ricketts). La position du point A est alors mise en relation avec la proximité radiculaire des incisives maxillaires. En effet, il y a souvent une confusion faite à tort entre position du point A et quantité d'os en regard des dents antérieures. Et de ce fait, une mauvaise évaluation de la quantité de déplacement possible au niveau de ces dents.

La téléradiographie de profil conventionnelle est encore aujourd'hui utilisée dans la majorité des cabinets pour le diagnostic, la conception du plan de traitement et l'évaluation des résultats d'un traitement. Cet article a pour objectif d'en évaluer la fiabilité pour quantifier l'épaisseur d'os alvéolaire en regard des incisives maxillaires. L'objectif second est de mettre en évidence un profil de patient dit «à risque » en évaluant les éventuelles corrélations entre cette épaisseur et des composantes de dysmorphie. Enfin, nous aborderons les conduites à tenir cliniquement pour éviter l'apparition des échecs parodontaux de type récessions gingivales, fenestrations et déhiscences osseuses dans ce secteur d'arcade.

\section{Fiabilité de la téléradiographie de profil quant à l'estimation de l'épaisseur de l'os alvéolaire en regard des incisives maxillaires [1]}

Un cliché radiographique en deux dimensions (2D) représente la projection d'un objet en trois dimensions (3D). Il en découle des problèmes liés au repérage des points (le point A ayant une reproductibilité comprise entre 1 et $2 \mathrm{~mm}$ selon l'étude de Lambert, et al. [5]) et des erreurs provenant d'une mauvaise interprétation des clichés, moins connues mais pouvant donner une fausse estimation aboutissant à des prises de risque dans les décisions thérapeutiques.

Sur la téléradiographie de profil, la distance séparant le point A des incisives maxillaires semble montrer une certaine « sécurité » dans la quantité d'os en regard de ces dents.

\subsection{Matériel et méthodes}

\subsection{1. Échantillon}

Léchantillon est formé de 50 sujets : 18 sujets de sexe masculin et 32 sujets de sexe féminin, âgés de 13,9 ans en moyenne (extrêmes : 7-54 ans).

Cet échantillon a été sélectionné à partir des 283 scanners disponibles dans la banque de donnée du logiciel Osirix ${ }^{\circledR}$ du cabinet d'orthodontie de Le Gall, Bachet et Dameron. Ont été inclus les scanners du maxillaire effectués dans les services de radiologie de la clinique de la Casamance ou de l'hôpital Edmond Garcin à Aubagne, d'une qualité suffisante et sans artéfacts, comprenant dans le champ d'irradiation au minimum la zone s'étendant de l'épine nasale antérieure jusqu'au bord libre des incisives maxillaires.

La présence des quatre incisives maxillaires était requise et le patient ne devait pas présenter d'éléments anatomiques venant perturber la zone étudiée (odontome, incisive incluse).

\subsubsection{Recueil de l'information}

Lépaisseur des coupes des examens scanner utilisés dans le cadre de l'étude est de 0,625 mm (scanners réalisés à la Casamance) et 0,5 mm (scanners réalisés à l'hôpital Edmond Garcin). Les données numériques des coupes natives sont enregistrées en format DICOM (Digital Imaging for Communication 
in Medecin). C'est un format universel permettant l'échange des données qui peuvent être exportées sur un support informatique adapté pour être traitées à l'aide du logiciel Osirix ${ }^{\circledR}$ (version v.5.8.5 32-bit système requis: MacOS 10.8 ou MacOS 10.9), permettant ainsi la production de l'imagerie et de la biométrie 3D des éléments anatomiques.

\subsection{Méthodes}

Dans un souci de simplification et de comparaison pertinente des mesures d'épaisseur d'os, nous avons choisi d'utiliser pour l'étude les céphalogrammes synthétisés à partir des données tomodensitométriques des patients.

En effet, le mode MIP (Maximum Intensity Projection) du logiciel Osirix ${ }^{\circledR}$ est un mode d'imagerie 2D en coupe épaisse qui nous a permis d'obtenir une superposition des structures tridimensionnelles sur une image en deux dimensions. Ainsi, on obtient un céphalogramme synthétisé qui peut s'apparenter à une téléradiographie de profil traditionnelle (Fig. 1).

Notons que d'autres études publiées dans la littérature utilisent cette technique et il a été démontré que des analyses céphalométriques 2D conventionnelles peuvent être réalisées avec précision sur ces céphalogrammes synthétisés, ne faisant apparaître aucune différence quant au repérage des points et aux mesures angulaires par rapport à ceux obtenus à partir de radiographies conventionnelles [2].

Sur chacun des 50 scanners, deux points ont été positionnés :

- Le point $A$ de Downs $\left(A_{D}\right)$ conformément à la définition de Downs : «Point le plus déclive sur la ligne médiane de la concavité du procès alvéolaire du maxillaire entre l'épine nasale antérieure et le prosthion.»

- Un second point situé sur le contour antérieur du rebord alvéolaire à la même hauteur que le point $A_{D}$ mais en regard de l'incisive la plus vestibulée et sur la coupe scanner centrée par rapport à la largeur mesio-distale de celle-ci. Ce nouveau point est appelé « Point A incisif » ou « Point $A_{I}$ ».

Deux types de mesures ont été effectués (en millimètre) (Fig. 3) :

- la distance du point $A_{D}$ à l'axe incisif;

- la distance du point $A_{D}$ à la surface radiculaire incisive (représente la mesure de l'épaisseur d'os estimée sur téléradiographie de profil);

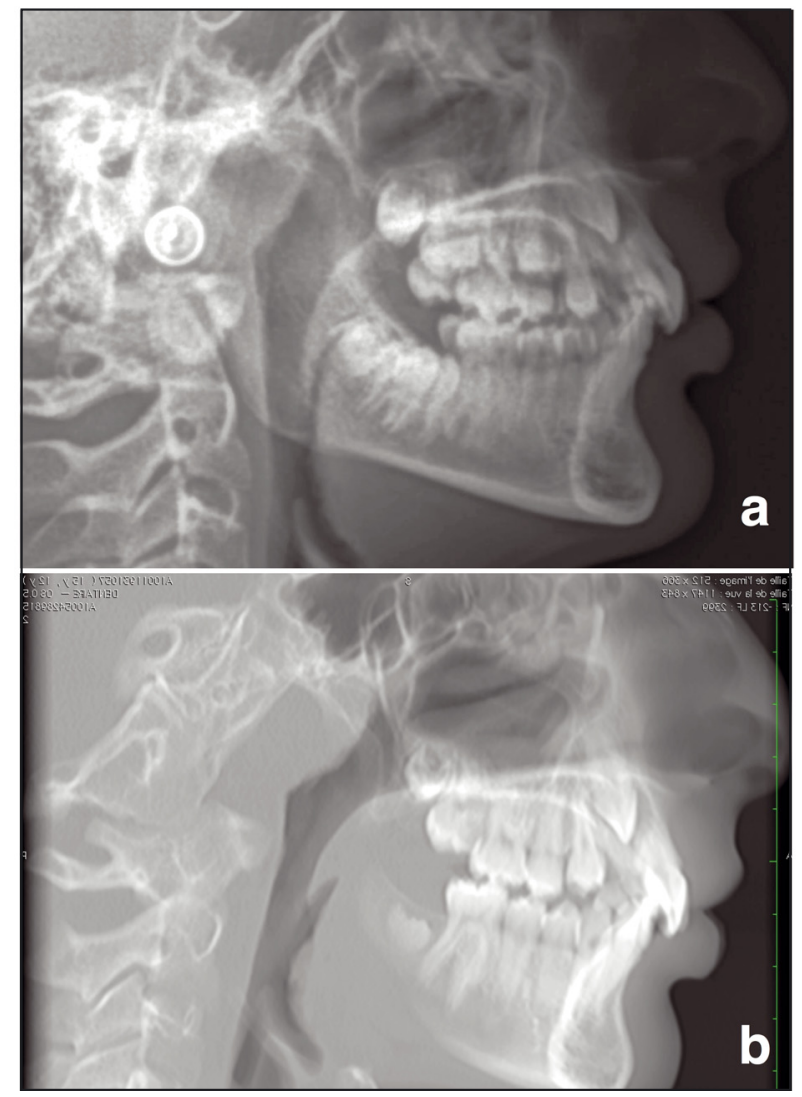

Figure 1

Céphalogrammes latéraux d'un même patient à partir d'une téléradiographie de profil conventionnelle (a) et obtenu à partir des données du scanner 2D en mode MIP moyen (épaisseur totale des coupes empilées $=31,06 \mathrm{~mm}$ ). Noter la ressemblance entre ces deux modes d'imagerie.

- la distance du point $\mathrm{A}_{\mathrm{I}}$ à l'axe incisif;

- la distance du point $A_{I}$ à la surface radiculaire incisive (représente la mesure de l'épaisseur d'os réelle).

Cette méthode (placement des points et mesures) a été répétée pour les 50 cas de l'étude selon le même protocole opératoire et par le même opérateur deux fois à quinze jours d'intervalle. Les mesures retenues pour l'étude représentent la moyenne des deux résultats effectués à quinze jours d'intervalle.

\subsection{Résultats}

La comparaison entre les distances « Point $A_{D}$ axe incisif » et «Point $A_{\mathrm{I}}$-axe incisif » révèle une différence en moyenne de 1,32 $\mathrm{mm}$ entre ces deux mesures.

La moyenne de l'épaisseur d'os estimée sur téléradiographie de profil est de 2,25 $\mathrm{mm}$ alors que 


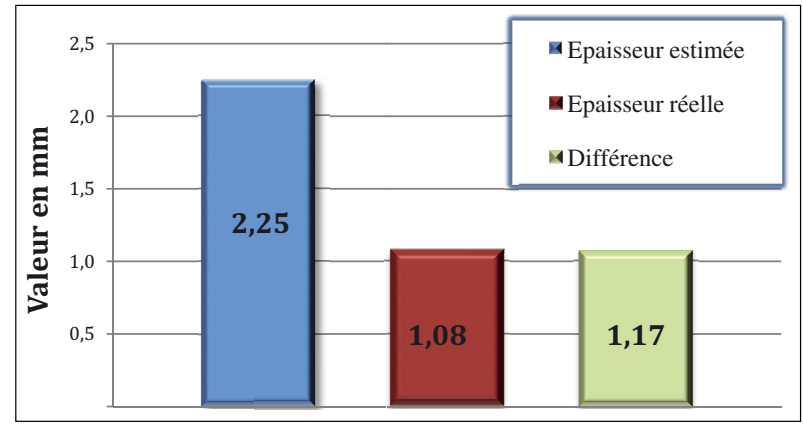

Figure 2

Moyennes et différence des moyennes entre l'épaisseur d'os alvéolaire estimée (TRP) et l'épaisseur d'os alvéolaire réelle (scanner) en regard des incisives maxillaires.

celle de l'épaisseur réelle mesurée sur les coupes 2D du scanner est de 1,08 $\mathrm{mm}$ soit une différence de $1,17 \mathrm{~mm}$

Ainsi, il existe une erreur de $50 \%$ dans l'estimation de la quantité osseuse en regard de l'incisive centrale maxillaires (Fig. 2)

La figure 3 présente le patient de l'étude pour lequel les valeurs maximales en termes de pourcentage d'erreur ont été relevées : 78 \% d'erreur d'estimation de l'épaisseur de l'os en regard des incisives maxillaires, soit une différence de 3,9 mm (Fig. 3).

Ainsi, la tomodensitométrie permet d'évaluer de façon plus précise la zone du point A par rapport à la téléradiographie de profil. Elle permet de mieux appréhender les reliefs anatomiques de cette zone, d'évaluer les rapports de proximité entre point A, incisives maxillaires et corticale osseuse et effectuer des mesures précises entre ces éléments. La téléradiographie de profil induit donc une erreur d'appréciation quant à l'épaisseur d'os alvéolaire présent en regard des incisives maxillaires.

\section{Corrélations entre épaisseur d'os alvéolaire et dysmorphies [1]}

\subsection{Matériel et méthodes}

Dans le but de dégager un profil de patient dit «à risque », nous avons étudié sur le même échantillon que l'étude précédente les corrélations éventuelles entre l'épaisseur d'os alvéolaire réel et les composantes de certaines dysmorphies.

Les 50 patients de l'étude présentaient tous dans leur dossier médical une téléradiographie de profil conventionnelle prise dans un délai de six mois maximum avant ou après l'acquisition du scanner. Les téléradiographies ont été prises sur le même céphalostat à 4,50 m. Elles ont été numérisées et importées dans le logiciel de céphalométrie en ligne « céphalométrics.fr » qui requiert l'installation du navigateur internet Google Chrome.

Les tracés céphalométriques ont été effectués à partir de l'analyse de Tweed et du CTTD et ont permis de classer les patients en :

- Normodivergent (FMA compris entre $20^{\circ}$ et $30^{\circ}$ );

- Hypodivergent $\left(\mathrm{FMA}<20^{\circ}\right)$;

- Hyperdivergent $\left(\mathrm{FMA}>30^{\circ}\right)$;

- Classe I squelettique (AoBo compris entre $-2 \mathrm{~mm}$ et $+2 \mathrm{~mm}$ ) ;

- Classe II squelettique (AoBo $>2 \mathrm{~mm}$ );

- Classe III squelettique (AoBo $<-2 \mathrm{~mm}$ );

- Inclinaison incisive «normale » avant traitement (I/NaA entre $18^{\circ}$ et $26^{\circ}$ );

- Vestibulo-version incisive avant traitement $\left(\mathrm{I} / \mathrm{NaA}>26^{\circ}\right)$;

- Palato-version incisive $\left(\mathrm{I} / \mathrm{NaA}<18^{\circ}\right)$.

\subsection{Résultats}

Les résultats du test de corrélation de Pearson entre épaisseurs d'os et typologie sagittale, verticale et orientation incisive sont présentés sous forme de graphique dans la figure 4.

Dans la dimension antéropostérieure, les malocclusions de classe I et II squelettique ne présentent pas une corrélation suffisamment importante pour établir une relation avec l'épaisseur d'os réelle. La malocclusion de classe III squelettique présente une corrélation positive qui commence à être significative $(+30,96)$. Ainsi, plus les valeurs de l'angle ANB diminuent (donc plus la classe III est prononcée), plus le risque de retrouver une épaisseur d'os réelle faible en regard des incisives maxillaires est important.

Dans la dimension verticale, les valeurs de corrélation obtenues dans les typologies normo et hypodivergentes ne permettent pas d'établir de conclusion quant à une éventuelle relation avec l'épaisseur d'os. En revanche, la typologie hyperdivergente semble être fortement corrélée à cette mesure. Cette corrélation est hautement négative $(-93,68)$ donc plus l'hyperdivergence est prononcée, plus le risque de retrouver une épaisseur d'os réelle faible en regard des incisives maxillaires est important. 


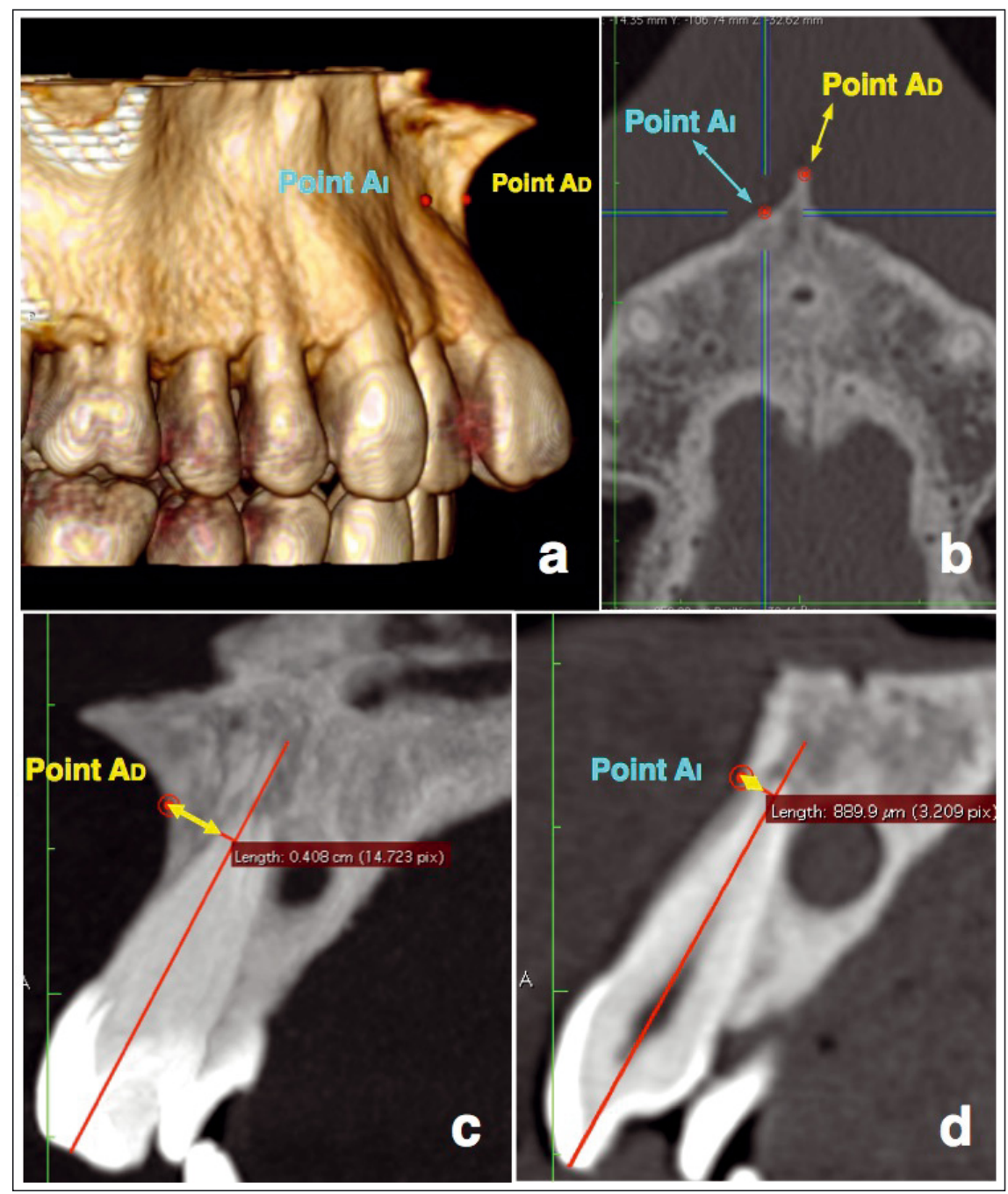

Figure 3

Cas extrême de l'étude : positionnement des points « $A$ de Downs $\left(A_{D}\right)$ et « $A$ incisif $\left(A_{I}\right)$ sur la vue $3 D$ (a) puis sur une coupe axiale (b). Mesure de l'épaisseur d'os estimée sur le céphalogramme latéral synthétisé en $2 \mathrm{D}=4,1 \mathrm{~mm}$ (c). Mesure de l'épaisseur d'os réelle = 0,9 $\mathrm{mm}$ (d). Soit une différence de 3,2 $\mathrm{mm}$ (78\% d'erreur d'estimation de la quantité d'os alvéolaire pour ce cas).

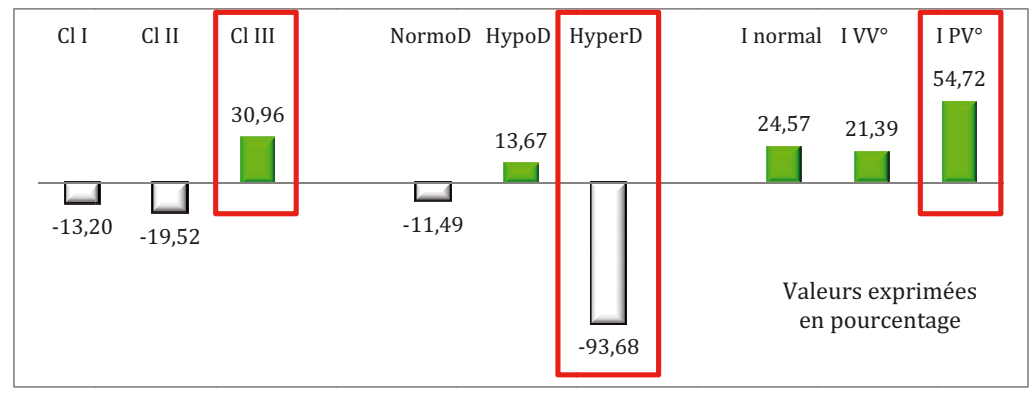

Figure 4

Corrélations entre épaisseur d'os réelle et typologie antéro-postérieure, verticale et orientation incisive maxillaire (en vert = corrélation positive; en blanc = corrélation négative). Les corrélations significatives sont encadrées en rouge. Pour une lecture plus facile du graphique, nous avons ramené ces résultats à un intervalle de -100 à +100 . On estime qu'à partir d'une corrélation de $|30|$ les variables sont liées et à partir de $|50|$ elles sont fortement liées. 
Concernant l'orientation initiale de l'incisive maxillaire, seule la variable «palatoversion» semble montrer une corrélation avec l'épaisseur d'os réelle. Cette corrélation étant positive $(+54,72)$, plus l'angle I/Na diminue (donc plus la palatoversion augmente) plus le risque de retrouver une épaisseur d'os réelle faible en regard des incisives maxillaires est important.

Remarque: Il serait intéressant de refaire ces calculs sur un échantillon beaucoup plus étendu que le nôtre. En effet, l'interprétation des tests de corrélation réalisés est à nuancer compte tenu du fait que, pour certaines variables, l'effectif était très faible.

\section{Applications cliniques : comment éviter l'échec parodontal dans la zone du point $A$}

La moyenne de l'épaisseur d'os alvéolaire en regard des incisives maxillaires obtenue dans notre étude, toute malocclusion confondue, est de $1,08 \mathrm{~mm}$.

Face à cette réalité clinique, objectivée par des mesures précises, nous devons tenir compte de ces observations et adapter notre thérapeutique.

\subsection{Respect des conditions du déplacement dentaire}

Il est admis que l'orthodontie n'a pas de conséquences délétères sur le parodonte lorsque ce dernier est sain ou assaini et que la dent se déplace non pas au travers, mais avec son os de soutien [3].

Pour Fontenelle, le modelage parodontal accompagnant le déplacement dentaire ne peut être obtenu raisonnablement que si trois conditions sont réunies:

- Conditions anatomiques : le parodonte doit être complet et sain.

- Conditions non inflammatoires: détartrage, surfaçage, chirurgie des poches.

- Conditions mécaniques : utilisation de forces légères et continues [6].

Malheureusement, il n'en est pas toujours ainsi et chaque fois que ces conditions ne sont pas respectées, la dent ne se déplacera pas avec mais au travers de son parodonte, créant récession gingivale, fenestration ou déhiscence osseuse. Une dent forcée contre une corticale fine ou absente peut ainsi créer une dysharmonie dento-parodontale par excès de matériel dentaire ou défaut de matériel osseux alvéolaire.

Dans le but de prévenir au maximum les conséquences parodontales délétères des traitements orthodontiques, l'orthodontiste doit avant tout traitement, en particulier chez l'adulte, analyser l'architecture parodontale du patient.

La classification de Maynard et Wilson décrivant quatre types de parodonte est un bon moyen d'apprécier le risque parodontal. Notre attention se portera principalement sur le type III (tissu kératinisé de dimension normale et épaisseur vestibulolinguale du procès alvéolaire mince) car il peut tromper la vigilance de l'orthodontiste qui devra faire très attention aux mouvements de vestibulo-version. Dans le type IV (tissu kératinisé $<2 \mathrm{~mm}$ et épaisseur vestibulo-linguale du procès alvéolaire mince), il existe un fort potentiel de récession si les conditions précitées du déplacement dentaire ne sont pas respectées [7].

\subsection{Examens d'imagerie 3D pour les cas à risque}

Comme développé dans la deuxième partie de notre étude, l'hyperdivergence, la classe III squelettique et une palato-version initiale de l'incisive maxillaire présentent une corrélation importante avec des épaisseurs très fines d'os alvéolaire en regard des incisives maxillaires.

Ainsi, pour ces cas à risque, des examens d'imagerie 3D peuvent se justifier puisqu'ils ne sont pas affectés des problèmes de projection dont souffrent les radiographies conventionnelles (Fig. 5).

Toutefois, l'impact dosimétrique de l'imagerie tridimensionnelle ne doit pas être perdu de vue et l'indication se justifie par un contexte clinique complexe. Nous ne pouvons qu'espérer dans l'avenir, avec les progrès technologiques, une diminution des doses d'irradiation pour que l'imagerie 3D se généralise.

\subsection{Remise en question de l'ancrage au point $A$}

Dans la technique du «Level Anchorage System », Root [8-10] utilisait le point A comme point d'ancrage pour aider la correction des « petites » classes II d'environ $3 \mathrm{~mm}$. Le point A servant d'ancrage, la consolidation des quatre incisives maxillaires par une chaînette élastomérique associée à la mise en 

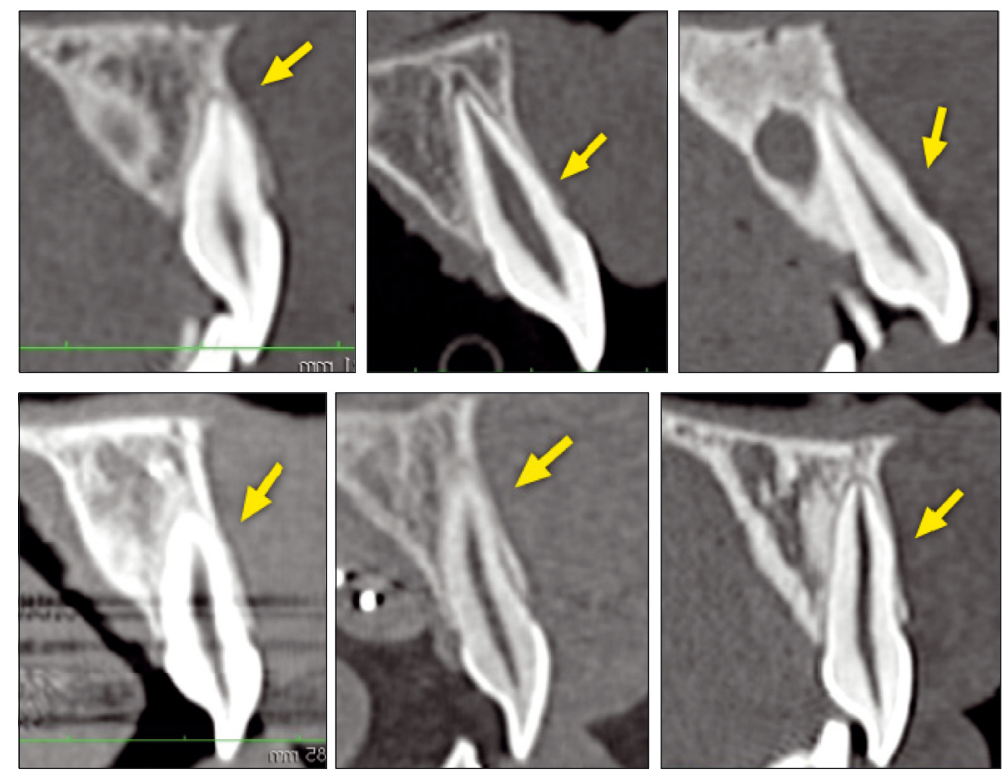

Figure 5

Coupes sagittales issues des examens tomodensitométriques de quelques uns des patients de l'étude. Notez l'extrême finesse de l'os alvéolaire en regard de l'incisive maxillaire.

place d'un fil 0,018 × 0,018 in NiTi sur une arcade baguée de 17 à 27 permet, d'après cette théorie, un mouvement distal des secteurs postérieurs.

Pour lui, la plupart des malocclusions de classe II ont un angle SNA presque normal et un angle SNB diminué. Idéalement, le point B devrait être avancé mais ce mouvement étant limité, il conseille de réduire SNA.

Il considère le point A comme la limite antérieure pour les racines supérieures. Comme le point B, selon lui, il peut être avancé, en avançant les racines.

Toujours pour cet auteur, dans les malocclusions de classe III, le point A fournit une zone d'ancrage pour une utilisation illimitée des élastiques de classe III. Il recommande l'utilisation d'un arc maxillaire pleine taille pour contrôler le torque des racines des dents antéro-supérieures lors de la mécanique de classe III [8-10].

Les résultats de notre travail permettent de remettre en question l'ancrage au point A et d'appliquer cette technique avec la plus grande prudence compte tenu de la faible valeur d'ancrage que peuvent fournir les dents et leur environnement osseux dans cette zone anatomique.

\subsection{Contrôle de l'axe incisif}

\subsubsection{Contrôle lors de l'alignement dentaire}

Lalignement dentaire sans précaution conduit à une expansion dans toutes les directions. Cet effet peut dans certains cas être recherché mais peut être néfaste dans d'autres, entrainnant les racines hors des alvéoles osseuses. Lorsque le contexte clinique le nécessite, il faut savoir contrôler l'axe incisif au cours de cette phase thérapeutique.

Par exemple, dans un cas nécessitant l'extraction de prémolaires, la présence d'un encombrement fait que les malpositions dans les zones antérieures de l'arcade provoqueront, si elles sont prises en charge immédiatement, une vestibuloversion des incisives pendant les phases d'alignement. En conséquence, il est préférable dans ces cas, de ne baguer en première intention que les dents les moins malpositionnées. Lalignement de l'arcade peut alors se faire avec un arc rectangulaire (super-élastique à mémoire de forme ou en acier tressé) permettant le contrôle de l'inclinaison des incisives et évitant ainsi une vestibuloversion qu'un fil rond engendrerait (Fig. 6).

Les techniques utilisant des boîtiers autoligaturants ne doivent pas inciter à moins extraire quand le cas le nécessite. Il ne faut pas repousser les limites physiologiques car une expansion non raisonnée peut provoquer une migration apicale de l'attache épithéliale et des dehiscences osseuses. 


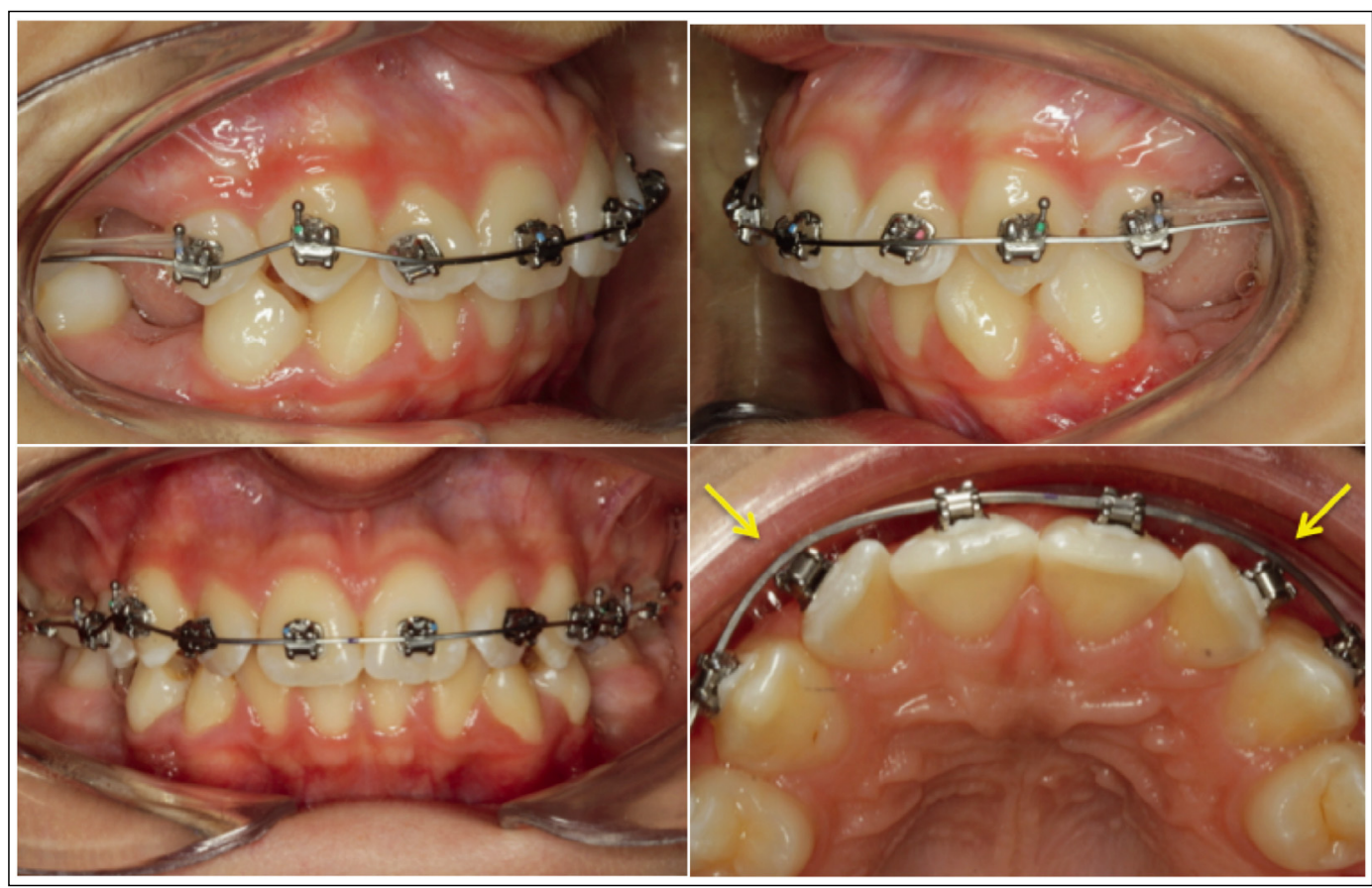

Figure 6

Jour du collage des attaches maxillaires après extractions des 15 et 25 . Les 12 et 22 présentant des rotations ne sont pas prises en charge en première intention. Système TGO, fil .019 ×.025 in à mémoire de forme, chaînette légère de recul des 14/24 pour désencombrer le secteur antérieur.

Cela est particulièrement vrai pour le secteur antérieur mais l'est aussi pour les secteurs latéraux. Dans les cas de corticale externe très fine, l'os cortical étant peu labile, les risques de destruction sans apposition de compensation sont importants et ces risques augmentent avec l'âge [6].

\subsubsection{Contrôle lors de la rétraction incisive}

La rétraction incisive est un mouvement favorable mais d'application délicate, car il nécessite un contrôle mécanique strict du rapport force/moment. Selon leur position initiale, les incisives doivent être rétractées avec un déplacement radiculaire plus ou moins important. Si le mouvement radiculaire doit être contrôlé, il est nécessaire d'incorporer du torque radiculo-palatin actif et tenir compte du jeu du fil dans le boîtier.

Le risque d'effets indésirables sur le parodonte peut être présent si la mécanique n'est pas adaptée et engendre des effets parasites tels que le "rabitting». Ce dernier peut apparaître en cas d'utilisation de fils ronds ou sous-dimensionnés, d'une force appliquée supérieure au moment ou encore en cas d'augmentation de la flexibilité de l'arc (en augmentant la longueur de fil, une boucle de rétraction augmente la flexibilité de l'arc).

Les changements qui se produisent au niveau de l'os alvéolaire ne peuvent pas être identifiés sur téléradiographie de profil mais sont clairement reconnus par tomodensitométrie.

Dans l'étude de Vardimon, et al. [11] sur les changements de l'épaisseur de l'os cortical vestibulaire pendant la rétraction des incisives maxillaires, lors de la rétraction avec version, le tiers apical de la racine de l'incisive centrale maxillaire se déplace antérieurement vers la corticale osseuse vestibulaire réduisant le volume osseux situé au dessus du point A de $19 \%$.

Par comparaison, lors de la rétraction avec torque, les volumes osseux vestibulaires au dessus et en dessous du point A augmentent tous les deux respectivement de $28 \%$ et de $65 \%$. Cela signifie que la partie apicale est au contraire déplacée postérieurement, loin de la corticale osseuse vestibulaire, ce qui diminue le risque de fenestration/déhiscence à ce niveau.

Les résultats de l'étude de Vardimon [11], déjà alarmants en termes de perte de volume osseux lors de la rétraction sans contrôle de l'axe incisif (perte 
de $19 \%$ ) sont minimisés par le fait que les mesures sont toutes faites sur des téléradiographies de profil et donc selon une épaisseur estimée et non réelle d'os en regard des incisives centrales maxillaires!

Rappelons que l'emploi d'un fil sous dimensionné ne permet pas de contrôler de façon optimale l'axe incisif au cours du mouvement de rétraction. Pour exemple, la prescription de torque sur les incisives centrales maxillaires en technique de Roth est de $+12^{\circ}$. Un fil de taille $0,016 \times 0,022$ inch inséré dans une gorge de $0,018 \times 0,025$ inch pour une valeur pré-informée de $+12^{\circ}$ de torque, on ne peut espérer obtenir que $4,8^{\circ}$ de torque effectif soit une perte de $7,2^{\circ}$ de contrôle de l'axe incisif (Source Dentaurum pour les valeurs de torque effectif).

Nous pouvons à ce stade souligner l'intérêt du système $\mathrm{TGO}^{\circledR}$ (technique de glissement optimisé) dont la particularité est d'avoir une prescription différente au niveau des attaches antérieures $(0,020 \times 0,028$ in $)$ et des attaches postérieures $(0,022 \times 0,028$ in $)$. Dans cette technique, la mise en place d'un fil $0,020 \times 0,025$ in lors de la phase de rétraction incisive maxillaire a pour avantages :

- un excellent contrôle dans la zone antérieure (torque totalement lu);

- une taille suffisante pour contrebalancer les effets parasites;

- une stabilité de la dimension transversale due à la rigidité de l'arc;

- un contrôle du $2^{\mathrm{e}}$ ordre de meilleure qualité;

- un meilleur glissement au niveau postérieur.

Utiliser des arcs pleine taille lors de la phase de rétraction incisive présente l'intérêt physiologique de répartir de façon égale les pressions sur toute la hauteur radiculaire contrairement au mouvement de rabitting dans lequel la pression est maximale à l'apex et au niveau du rebord alvéolaire (Fig. 7).

\subsubsection{Contrôle lors de la mécanique inter-arcade}

La mécanique inter-arcade non contrôlée présente également un risque de détérioration du support osseux en regard des incisives maxillaires. C'est pourquoi les précautions en terme de contrôle d'axe doivent également être appliquées lors de cette phase thérapeutique.

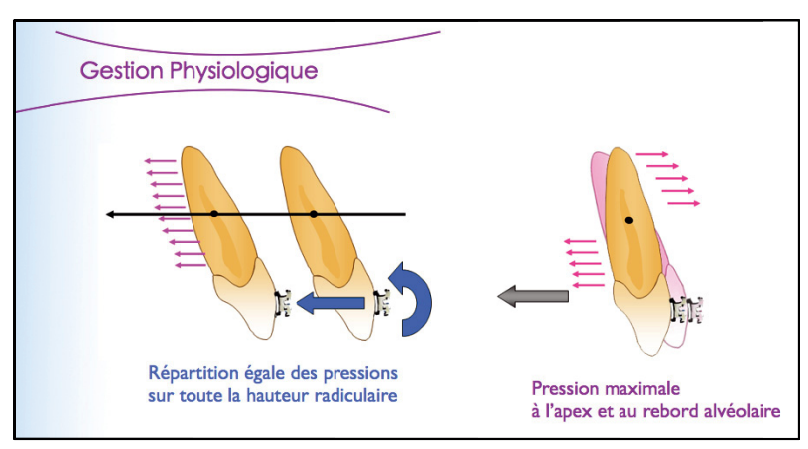

Figure 7

Gradient de pression avec et sans contrôle de l'axe incisif au cours de la phase de rétraction. La répartition des pressions sur toute la hauteur radiculaire permet une gestion plus physiologique du déplacement dentaire.

\section{Conclusion}

La plupart des techniques orthodontiques focalisent leur attention sur le contrôle de l'incisive mandibulaire et ne prennent pas de précautions particulières quant au déplacement de l'incisive maxillaire. Or, la téléradiographie de profil induit une erreur d'appréciation de moitié quant à l'épaisseur d'os alvéolaire présent en regard des incisives maxillaires.

Ce pourcentage d'erreur est donc à prendre en considération lors de l'établissement du plan de traitement orthodontique et particulièrement chez les patients présentant une hyperdivergence marquée, une classe III et dans une moindre mesure une palatoversion incisive.

La mise au point de l'imagerie 3D permet d'échapper aux contraintes liées aux caractéristiques bidimensionnelles des radiographies conventionnelles, offrant la possibilité de réaliser tout type de mesures avec une précision de l'ordre du dixième de millimètre, enrichissant et complétant le diagnostic orthodontique.

Un traitement orthodontique bien mené se doit d'obtenir un alignement dentaire optimal dans un contexte esthétique et fonctionnel. Le positionnement radiculaire est souvent mis de côté dans la mesure où l'appréciation de la situation radiculaire nécessite des examens d'imageries qui ne peuvent être renouvelés trop souvent et cela peut engendrer à plus ou moins longs termes des échecs parodontaux [4].

Avec l'avènement des scanners et du cone beam, les modélisations intégrant la morphologie radiculaire et l'environnement osseux aux critères de fin de traitement sont en train de se développer. Lindividualisation du traitement orthodontique qui existe 
déjà dans certaines techniques va s'accroître dans les prochaines années et la possibilité de visualiser les racines dans la position souhaitée à la fin du traitement est un outil qui va s'avérer précieux pour l'orthodontiste.

Néanmoins, c'est la connaissance et la compréhension des règles de la physiologie et de la biomécanique du déplacement dentaire qui permettent au praticien d'anticiper certains pièges et d'éviter les échecs parodontaux. Et ce n'est pas parce que la réalité clinique de nos actes n'est pas toujours visible qu'il faut la nier et ne pas la rechercher. Au contraire, il faut s'aider des recherches actuelles pour améliorer nos moyens diagnostiques afin d'anticiper l'apparition de séquelles dentaires et parodontales préjudiciables et irréversibles.

\section{Conflit d'intérêts}

Les auteurs déclarent n'avoir aucun lien d'intérêt concernant les données publiées dans cet article.

\section{Bibliographie}

[1] Chevalier E. Évaluation comparative de la zone du point A sur téléradiographie du crâne de profil et sur scanner : applications cliniques. Mémoire CECSMO. Marseille : Université d'Aix-Marseille, 2014.
[2] Chung RR, Lagravere MO, Flores-Mir C, Heo G, Carey JP, Major PW. Analyse comparative des valeurs céphalométriques de céphalogrammes latéraux générés par CBCT versus céphalogrammes latéraux conventionnels. Int Orthod 2009;7:308-321.

[3] Fontenelle A. Une conception parodontale du déplacement dentaire provoqué : évidences cliniques. Rev Orthop Dentofac 1982;16:37-53.

[4] Goglin-Benoit A, Jaisson M, Palot C, Barthelemi S. Anticipating root displacements : unlocking new prospects. Int Orthod 2011;9:286-297.

[5] Lambert A, Setbon O, Salmon B, Sebban V. Analyse céphalométrique. Encycl Méd Chir, Odontologie/Orthopédie dentofaciale, Paris: Elsevier; 2010, 23455-D-10.

[6] Massif L, Frapier L. Orthodontie et parodontie. Encycl Méd Chir, Odontologie/Orthopédie dentofaciale, Paris: Elsevier; 2007, 23-490-A-7

[7] Maynard JG, Wilson RD. Diagnosis and management of mucogingival problems in children. Dent Clin North Am 1980;24:683-703.

[8] Root TL. The level anchorage system for correction of orthodontic malocclusions. Am J Orthod 1981;80:395-410.

[9] Root TL. Concepts d'ancrage et finition des cas orthodontiques. J Edg 1980;1:11-29.

[10] Root TL. Level anchorage system treatment of Class III malocclusions. Am J Orthod Dentofac Orthop 1991;100:562-571.

[11] Vardimon AD, Oren E, Ben-Bassat Y. Cortical bone remodeling/tooth movement ratio during maxillary incisor retraction with tip versus torque movements. Am J Orthod Dentofac Orthop 1998;114:520-529. 